\title{
Outcomes of patients admitted to the intensive care unit for complications of hypertensive disorders of pregnancy at a South African tertiary hospital - a 4-year retrospective review
}

\author{
S Gama, ${ }^{1}$ MB BCh, FRCA; M Sebitloane, ${ }^{2}$ MB ChB, FCOG, MMed (Obstetr Gynaecol), PhD; OrcID 0000-0003-4046-0653; \\ K de Vasconcellos, ${ }^{1} \mathrm{MB}$ ChB, DA (SA), FCA (SA), Cert Crit Care (SA); OrcID 0000-0002-1974-6633 \\ ${ }^{1}$ Department of Anesthesiology and Critical Care, Nelson R Mandela School of Medicine, University of KwaZulu-Natal, Durban, South Africa \\ ${ }^{2}$ Department of Obstetrics and Gynaecology, Nelson R Mandela School of Medicine, University of KwaZulu-Natal, Durban, South Africa
}

Corresponding author: S Gama (nsgama2@aol.com)

\begin{abstract}
Background. Hypertensive disorders of pregnancy (HDP) are a major cause of maternal mortality and adverse outcomes. A previous study in the intensive care unit (ICU) at King Edward VIII Hospital, Durban, South Africa, in 2000 found 10.5\% mortality among eclampsia patients.

Objectives. To describe the mortality and adverse neurological outcomes associated with HDP in a tertiary ICU, compare these with results from 2000 and describe factors associated therewith.

Methods. The data of 85 patients admitted with HDP to ICU at King Edward VIII Hospital from 2010 to 2013 were retrospectively reviewed. Mortality and adverse neurological outcome (Glasgow Coma Scale (GCS) $\leq 14$ on discharge from ICU) were assessed. Two sets of analyses were conducted. The first compared those alive on discharge from ICU with those who died in ICU. The second compared good neurological outcome with poor outcome (adverse neurological outcome, or death).

Results. The mortality was $11.6 \%$, and overall, $9 \%$ had adverse neurological outcomes. There was no significant difference in mortality between patients with eclampsia in 2010 - $2013(11.0 \%)$ and those in $2000(10.5 \%)(p=0.9)$. Factors associated with mortality were: intra- or postpartum onset of seizures; twins; failure to perform operative delivery when indicated; lowest GCS score <10; failure to use magnesium sulphate when indicated; respiratory failure; and lower respiratory tract infections. Factors associated with poor outcomes (adverse neurological outcome, or death) were: parity (better outcomes in primiparous patients); time of antenatal onset of hypertension (worse if earlier onset); HIV infection; failure to perform operative delivery when indicated; lowest GCS score <10; failure to use magnesium sulphate when indicated; use of anticonvulsants other than magnesium sulphate or benzodiazepines in eclampsia.

Conclusion. The lack of improvement in ICU eclampsia mortality demonstrates a need to develop and implement a protocol for HDP management. Keywords. Hypertensive disorders of pregnancy, intensive care unit, mortality, neurological outcomes, eclampsia, human immunodeficiency virus.
\end{abstract}

S Afr J Crit Care 2019;35(2):62-69. https://doi.org/10.7196/SAJCC.2019.v35i2.001

\section{Contribution of the study}

- The study provides a comparison of present mortality among eclamptic patients with hyperensive disorders of pregnancy (HDP) with the mortality of eclamptic patients described in an article from the year 2000. It further looks at adverse maternal outcomes, specifically adverse neurological outcomes.

- In addition, it analyses other factors that may affect outcomes in HDP patients. This information is useful in making recommendations in an attempt to improve the outcomes.

Hypertensive disorders of pregnancy (HDP) are among the leading causes of maternal mortality in South Africa (SA) and worldwide. ${ }^{[1-3]}$ Various definitions and classification systems have been produced; however, the most widely used is that of the International Society for the Study of Hypertension in Pregnancy. ${ }^{[4]}$

The classification was updated in 2014 and now encompasses chronic hypertension, gestational hypertension, pre-eclampsia (de novo or superimposed onto chronic hypertension), and white-coat hypertension. ${ }^{[5]}$ Gestational hypertension and pre-eclampsia have their onset after 20 weeks. Pre-eclampsia is differentiated from gestational hypertension by the presence, in addition to hypertension, of proteinuria, other maternal organ dysfunction (renal, liver and/or haematological) and uteroplacental dysfunction (fetal growth restriction).

The pathogenesis of HDP is not fully understood, but it is thought that immune-genetic factors result in endothelial abnormalities. ${ }^{[6]}$ These abnormalities are mediated by factors that include cytokines, lipid peroxidation and fragmented fibronectin. The abnormal endothelium results in widespread vasoconstriction. This is due to the failure of the endothelium to produce adequate prostacyclin and possibly nitric oxide, coupled with an increase in platelet-derived factors such as thromboxane and serotonin. The abnormal endothelium also causes acceleration of the procoagulant state of pregnancy. 
In early placentation, there is failure of spiral artery dilatation, and placental ischaemia follows. ${ }^{[6]}$ Fetal intrauterine growth restriction and reproductive failure can occur. The vasoconstriction further results in hypertension. Other organ systems are affected, including the kidneys, brain and liver. Clinical manifestations include proteinuria, eclampsia (seizures) and haemolysis, elevated liver enzymes and low platelets (HELLP) syndrome. The clinical history can be one of indolent progression or rapidly progressive multi-organ disease.

It has been previously shown that care in an intensive care unit (ICU) reduces mortality in patients with eclampsia. ${ }^{[7,8]} \mathrm{A}$ previous study conducted in the ICU at King Edward VIII Hospital (Durban) in 2000 found a mortality rate of $10.5 \%{ }^{[9]}$ The study also demonstrated that the organ system failure and Glasgow Coma Scale (GCS) scores were good predictors of outcome in eclampsia.

The present study aimed to describe the outcomes of HDP patients managed in the same ICU in 2010 - 2013.

Researchers have suggested maternal morbidity as an important category to study distinctly from mortality ${ }^{[8,10]} \mathrm{A}$ widely used definition of maternal morbidity defines a 'near miss' as a situation in which a patient has an acute organ system dysfunction that if not treated appropriately could have resulted in death. ${ }^{[1,12]}$ This definition was formulated in a first-world context. It was reasoned that maternal mortality numbers were too small to enable an adequate assessment of disease patterns among obstetrics patients, and to therefore make recommendations that would be relevant to the care of most obstetrics patients. Audits of maternal morbidities or near misses were therefore recommended. The clinical criteria for morbidity were organ dysfunction (cardiac, respiratory and others) or management based. Importantly for this study, the management-based criteria included ICU admission, for any reason. Any ICU-based study of obstetrics patients is especially relevant, as all cases by definition are morbidities. It is, however, important to note that they are only a subset of maternal morbidities.

For the purposes of this study, adverse maternal outcome was assessed, here referring to the neurological condition of the patient on discharge from ICU. The GCS was used because it is the most commonly used in the acute clinical setting in HDP. However, it has also been found that maternal cognitive impairment may last several months or be permanent after severe pre-eclampsia or eclampsia. ${ }^{[13]}$

\section{Methods \\ Study design}

The present study was a retrospective analysis of patients admitted to a tertiary centre ICU with HDP. It used a retrospective chart review of patients admitted to ICU with HDP in 2010 - 2013, with the aim of describing the mortality and adverse neurological outcomes associated with HDP, and comparing these with results from a study ${ }^{[9]}$ conducted in the same hospital in 2000. Additionally, the factors that may have contributed to any improvement or deterioration in the mortality rate were evaluated. The factors associated with mortality were: intra- or postpartum onset of seizures; twins; failure to perform operative delivery when indicated; lowest GCS score $<10$; failure to use magnesium sulphate when indicated; respiratory failure; and lower respiratory tract infections. Factors associated with poor outcomes (adverse neurological outcome, or death) were: parity (better outcomes in primiparous patients); time of antenatal onset of hypertension (worse if earlier onset); HIV infection; failure to perform operative delivery when indicated; lowest GCS score $<10$; failure to use magnesium sulphate when indicated; use of anticonvulsants other than magnesium sulphate or benzodiazepines in eclampsia.

All data were obtained from existing patient records. The data collected were: general data (Tables 1 and 2); neurological findings and

Table 1. General and antenatal data: alive (neurologically intact or adverse neurological outcome) v. dead ${ }^{*}$

\begin{tabular}{|c|c|c|c|c|c|c|}
\hline Variable & $\begin{array}{l}\text { All patients, } \\
\text { median (IQR) }\end{array}$ & Alive, $n(\%)$ & $\begin{array}{l}\text { Alive, median } \\
\text { (IQR) }\end{array}$ & Dead, $n(\%)$ & Dead, median (IQR) & $p$-value \\
\hline \multicolumn{7}{|l|}{ General data } \\
\hline Age (years) $(N=82)$ & $22(19-27)$ & $72(87.8)$ & $(22(19-27))$ & $10(12.2)$ & $26(25-33)$ & 0.055 \\
\hline ICU length of stay (days) $(N=81)$ & $4(3-5)$ & $73(90.1)$ & $4(3-5)$ & $8(9.9)$ & $4(2-5)$ & 0.798 \\
\hline Parity $(N=76)$ & & & & & & 0.72 \\
\hline Primiparous $(n=41)$ & - & $39(95.1)$ & - & $2(4.9)$ & - & \\
\hline $\begin{array}{l}\text { Time of antenatal onset of hypertension } \\
\text { (weeks) }(N=57)\end{array}$ & $33(28-36)$ & $51(89.5)$ & $33(29-36)$ & $6(10.5)$ & $28(26-36)$ & 0.283 \\
\hline \multicolumn{7}{|l|}{ Management at ANC } \\
\hline \multicolumn{7}{|l|}{ Booked $\geq 2$ visits $(N=56)$} \\
\hline No $(n=12)$ & - & $11(91.7)$ & - & $1(8.3)$ & - & 0.506 \\
\hline Yes $(n=44)$ & - & $37(84.1)$ & - & $7(15.9)$ & - & \\
\hline \multicolumn{7}{|l|}{ Risk factors for HDP } \\
\hline No $(n=29)$ & - & $23(79.3)$ & - & $6(20.7)$ & - & 0.066 \\
\hline Yes $(n=56)$ & - & $52(92.9)$ & - & $4(7.1)$ & - & \\
\hline \multicolumn{7}{|l|}{ Primiparous $(N=76)$} \\
\hline No & - & $28(80.0)$ & - & $7(20.0)$ & - & 0.083 \\
\hline Yes & - & $39(95.1)$ & - & $2(4.9)$ & - & \\
\hline \multicolumn{7}{|l|}{ Twins $(N=81)$} \\
\hline No & - & $69(90.8)$ & - & $7(9.2 \%)$ & - & $0.034^{\dagger}$ \\
\hline Yes & - & $3(60.0 \%)$ & - & $2(40.0 \%)$ & - & \\
\hline $\begin{array}{l}\mathrm{IQR}=\text { interquartile range; } \mathrm{ICU}=\text { intensive care unit; } \mathrm{A} \\
{ }^{*} N \text { varies as patients with missing data were excluded. } \\
{ }^{t} p=0.05 \text {. }\end{array}$ & enatal clinic; $\mathrm{HD}$ & pertensive disor & ers of pregnancy. & & & \\
\hline
\end{tabular}




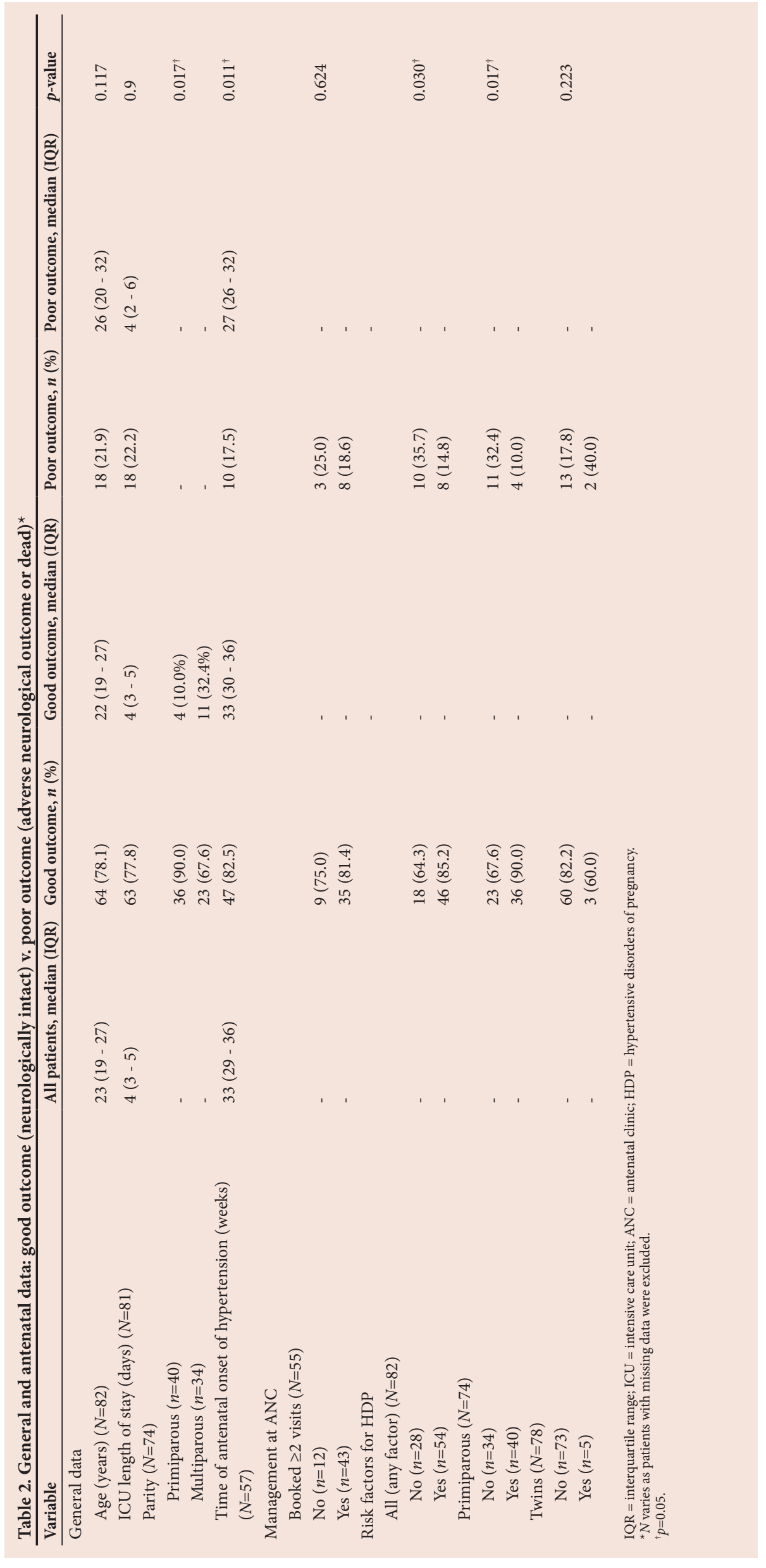

use of anticonvulsants (Tables 3 and 4); and data on critical care findings (Tables 5 and 6). The primary outcomes of interest were mortality and adverse neurological outcome (defined as a GCS score $\leq 14$ on discharge from ICU). In addition, the impact of other factors on mortality and on adverse neurological outcome was assessed.

Patients were divided into three groups according to their outcome on discharge from ICU: those who were alive and neurologically intact (intact consciousness (GCS 15/15) and motor and sensory function); those who had an adverse neurological outcome; and those who had died. Two sets of analyses were conducted to determine whether any of the variables made a significant difference to the outcome. The first compared those who were alive (the neurologically intact, and those who had an adverse neurological outcome on discharge from ICU) with those who had died (Tables 1, 3 and 5). The second analysis compared patients who had a good outcome (neurologically intact on discharge from ICU) with those who had a poor outcome (those with an adverse neurological outcome, and those who had died) (Tables 2, 4 and 6).

Statistical analysis was conducted to determine whether each of the factors made a difference to outcome. Approval for the study was obtained from the University of KwaZulu-Natal Biomedical Research Ethics Committee (ref. no. BE006/14), King Edward VIII Hospital and the Health Research Committee of the KwaZuluNatal Department of Health (ref. no. HRKM 112/14). ICU patients should have two sets of notes, from which the data were extracted: ward notes and ICU notes (including ICU admission notes, daily notes and discharge summaries). Fig. 1 describes the sources used to extract data and the inclusion criteria for the study.

\section{Statistical methods}

Statistical analyses were performed using the Statistical Product and Service Solution (SPSS) version 23.0 (IBM Corp., USA). Categorical variables were described as percentages and compared using the $\chi^{2}$ test or Fisher's exact test. Continuous, normally distributed data were described using means (standard deviations) and analysed using analysis of variance and Student's $t$-test. Non-parametric data were described using median and interquartile 
Table 3. Neurological findings: alive (neurologically intact or adverse neurological outcome) v. dead*

\begin{tabular}{|c|c|c|c|c|}
\hline Variable & Total, $n$ & Alive, $n(\%)$ & Dead, $n(\%)$ & $p$-value \\
\hline \multicolumn{5}{|l|}{ Eclampsia $(N=81)$} \\
\hline No & 27 & $24(88.9)$ & $3(11.1)$ & \multirow[t]{2}{*}{0.702} \\
\hline Yes & 54 & $48(88.8)$ & $6(11.1)$ & \\
\hline \multicolumn{5}{|l|}{ Lowest recorded GCS $(N=76)$} \\
\hline$<10$ & 36 & $28(77.8)$ & $8(22.2)$ & \multirow[t]{2}{*}{$0.011^{\dagger}$} \\
\hline$\geq 10$ & 40 & $39(97.5)$ & $1(2.5)$ & \\
\hline \multicolumn{5}{|l|}{ CT scan findings } \\
\hline \multicolumn{5}{|l|}{ CT scan done $(N=80)$} \\
\hline No & 48 & 45 (93.8) & $3(6.2)$ & \multirow[t]{2}{*}{0.08} \\
\hline Yes & 32 & $25(78.1)$ & $7(21.9)$ & \\
\hline \multicolumn{5}{|l|}{ Abnormal CT findings $(N=32)$} \\
\hline No & 12 & $10(83.3)$ & $2(16.7)$ & \multirow[t]{2}{*}{0.581} \\
\hline Yes & 20 & $15(75.0)$ & $5(25.0)$ & \\
\hline \multicolumn{5}{|l|}{ CT structural damage $(N=32)$} \\
\hline No & 21 & $18(85.7)$ & $3(14.3)$ & \multirow[t]{2}{*}{0.151} \\
\hline Yes & 11 & $7(63.6)$ & $4(36.4)$ & \\
\hline \multicolumn{5}{|l|}{ Emergency anticonvulsants } \\
\hline \multicolumn{5}{|c|}{ Magnesium sulphate (severe pre-eclampsia and eclampsia) $(N=80)$} \\
\hline \multicolumn{5}{|c|}{ No } \\
\hline Yes & 20 & $14(70.0)$ & $6(30.0)$ & \multirow[t]{3}{*}{$0.006^{+}$} \\
\hline Magnesium sulphate (eclampsia) $(N=51)$ & 60 & $57(95.0)$ & $3(5.0)$ & \\
\hline No & 5 & $3(60.0)$ & $2(40.0)$ & \\
\hline Yes & 46 & $43(93.5)$ & $3(6.5)$ & 0.069 \\
\hline \multicolumn{5}{|l|}{ Benzodiazepines (eclampsia) $(N=51)$} \\
\hline No & 36 & $34(94.4)$ & $2(5.6)$ & \\
\hline Yes & 15 & $12(80.0)$ & $3(20.0)$ & 0.144 \\
\hline \multicolumn{5}{|l|}{ Other anticonvulsants (eclampsia) $(N=51)$} \\
\hline No & 46 & $42(91.3)$ & $4(8.7)$ & \\
\hline Yes & 5 & $4(80.0)$ & $1(20.0)$ & 0.416 \\
\hline
\end{tabular}

range (IQR), and analysed using the Kruskal-Wallis and Mann-Whitney $U$ tests.

\section{Results}

Over the 4-year period from 2010 to 2013 , there were 231 obstetrics patients admitted to ICU, out of a total of 1528 ICU admissions (15.1\%). Of these, 102 (44\% of obstetrics admissions) had HDP-related complications, the data of 85 of whom were analysed. Neurological outcome was unknown in three patients $(n=3 / 85)$ who were alive on discharge from ICU.

There were 55 patients with eclampsia and 25 with other HDP complications, which included hypertensive urgency and pulmonary oedema. Five patients could not be classified in terms of eclampsia, owing to missing data.

\section{Mortality}

There were 10 deaths, giving an overall mortality rate of $11.6 \%$. The mortality rate was the same (11.1\%) in eclampsia patients $(n=6 / 54)$ and non-eclampsia patients $(n=3 / 27)$.

\section{Other adverse maternal outcomes}

Ten patients had HDP-related adverse maternal outcomes on discharge from ICU. One patient had cardiac failure, and another had acute kidney injury requiring dialysis. These two patients were not included in the analysis of other adverse maternal outcomes owing to their small number. Of note, in $n=8 / 10(80 \%)$ of those with adverse maternal outcomes on discharge from ICU, the adverse outcomes were neurological (5 (9.1\%) eclampsia patients and $2(8 \%)$ non-eclampsia patients; in 1 patient it was unknown whether she had eclampsia). The overall adverse neurological outcome rate was $8.99 \%$. Therefore, only adverse neurological outcome was used in the analysis of adverse maternal outcome in this study.

When patients who were alive on discharge were compared with those who died, several factors were found to have a significant effect on this outcome. These were general factors (intra- or postpartum onset of seizures; twin pregnancy) (Table 1); comorbidities (failure to perform operative delivery when indicated - see supplementary data (http://sajcc.org.za/public/sup/sajcc_001.docx)); neurological findings and anticonvulsants (lowest GCS score $<10$, failure to use magnesium sulphate when it was indicated) (Table 3); and critical care findings (respiratory failure, lower respiratory tract infections) (Table 5).

When patients with a good outcome were compared with those who had a poor outcome on discharge from ICU, a different set of factors were found to have made a significant difference. These were:

- parity (better outcomes for primiparous patients; $p=0.017$ ) (Table 2)

- time of antenatal onset of hypertension (worse outcomes for those with earlier onset; $p=0.011$ ) (Table 2)

- comorbidities such as HIV (better outcomes among those without HIV infection; $p=0.017$ ); see supplementary data (http://sajcc.org.za/ public/sup/sajcc_001.docx)

- failure to perform operative delivery when indicated (performed less often in those with poor outcomes; $p=0.009$ ) (see supplementary data) - neurological findings (eclampsia; lowest GCS score <10) (Table 4) 


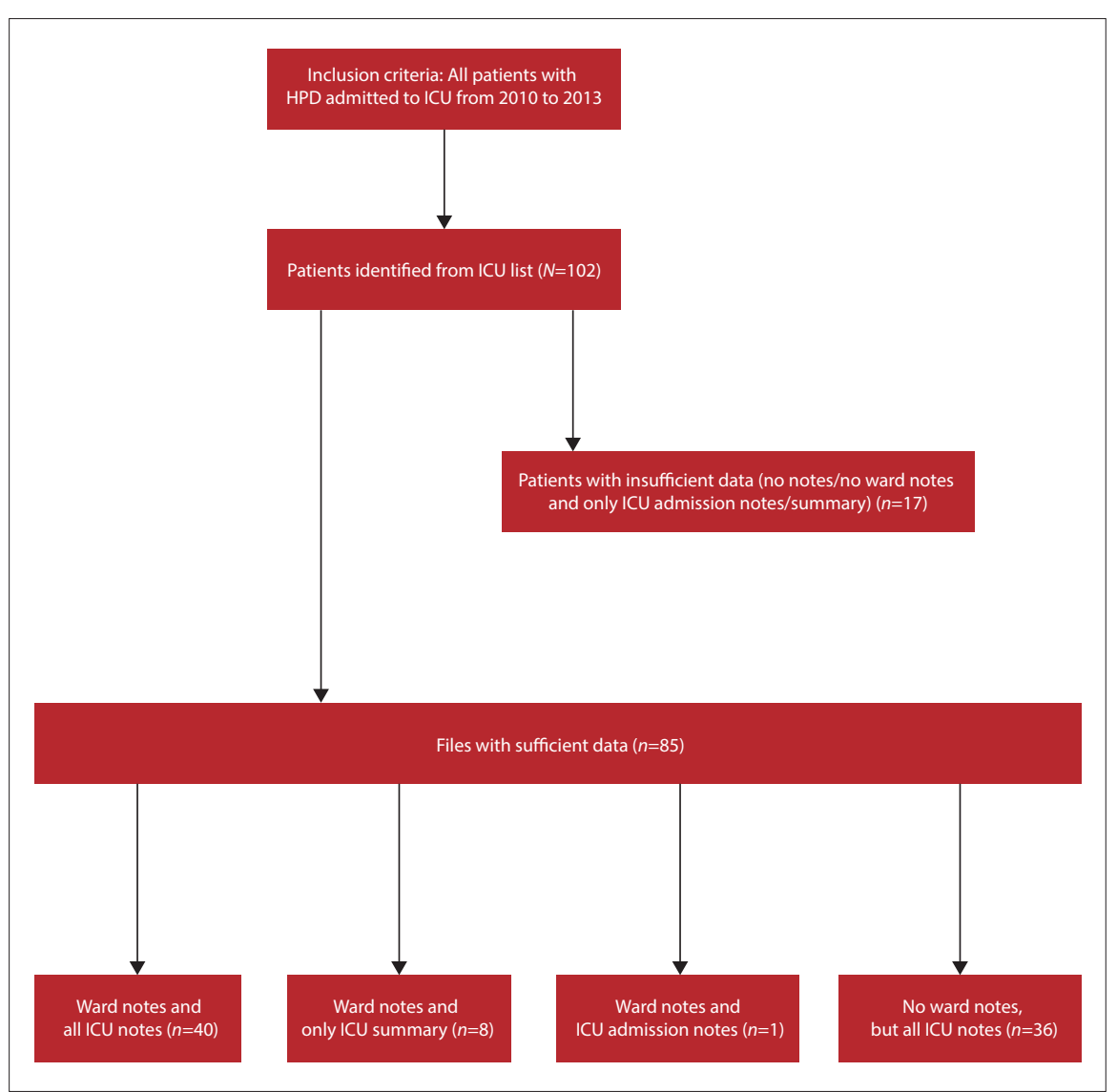

Fig. 1. Sources of patient information. Ward notes were obtained from the Medical Records Department of King Edward VIII Hospital; ICU notes were obtained from ICU. (HDP = hypertensive disorders of pregnancy; ICU = intensive care unit.)

- failure to use magnesium sulphate when it was indicated (used less in those with poor outcomes; $p=0.003$ ) (Table 4 )

- need for use of anticonvulsants other than magnesium sulphate or benzodiazepines in eclampsia (used more in those with poor outcomes; $p=0.048$ ) (Table 4 ).

\section{Discussion}

Among eclamptic patients admitted to ICU, the mortality during 2010 - 2013 was similar to that found by Bhagwanjee et al. ${ }^{[9]}$ in 2000 ( $11 \%$ and $10.5 \%$, respectively). In addition, the present analysis showed a significant proportion of patients who were alive on discharge but had adverse maternal outcome (8.99\%) (Tables 2, 4, 6 and supplementary data).

Compared with the year 2000, the absolute number of annual ICU mortalities due to these disorders decreased, as the number of annual ICU admissions with HDP has decreased. The reason for the decrease in the absolute number of HDP patients admitted to ICU has not yet been determined.

The lack of difference in outcomes (by proportion) between the year 2000 and the years 2010 - 2013 indicates a need for measures to decrease the rate of poor outcomes.
It was previously recommended that closer attention to neurological management may be beneficial. ${ }^{[9]}$ Some neurological factors showed significant differences in outcome in both analyses (Tables 3 and 4). The effect of the GCS score on outcome has been pointed out previously. ${ }^{[7,9,13-15]}$ A lowest GCS score of $\leq 10$ was associated with increased poor outcome and mortality. In our study, the when the patients were not immediately postictal, and were not being actively sedated.

In our setting, management of HDP patients requiring ICU, including eclamptic patients, did not include routine computed tomography (CT) scanning of the brain. Zeeman ${ }^{[13]}$ has recommended brain CT scans in patients with focal neurological signs, atypical or recurrent convulsions, prolonged coma and prolonged return to complete recovery following delivery. It is probably left to clinical judgement how long precisely 'prolonged' recovery of consciousness after an eclamptic seizure should be.

The role of neurosurgery (in patients with intracranial haemorrhage) should also be explored. Another important recommendation lowest GCS scores used were those assessed is rapid treatment of seizures, and close attention to neuroprotective measures while the patients are intubated and being ventilated. ${ }^{[16]}$ Neuroprotective measures include adequate cerebral perfusion pressures, oxygenation, ventilation (arterial carbon dioxide control), glucose control and head elevation. ${ }^{[17]}$

A factor that influenced outcomes was failure to use magnesium sulphate where and when it was appropriate. The importance of magnesium sulphate is shown by the fact that there were poorer outcomes in those who were not given it when appropriate. Magnesium sulphate therapy for eclampsia was largely established after the Collaborative Eclampsia Trial was published in 1995. ${ }^{[18]}$ The trial found lower rates of seizure recurrence among patients given magnesium sulphate for eclamptic seizures than those treated with diazepam or phenytoin. Subsequently, magnesium sulphate prophylaxis in severe pre-eclampsia gained widespread acceptance ${ }^{[4,19]}$ after the Magpie Trial in 2002, which demonstrated lower rates of eclampsia if magnesium sulphate was used (compared with placebo) as seizure prophylaxis in eligible pre-eclamptic patients. ${ }^{[20]}$ Hence it is used in all eclamptic and severe pre-eclamptic patients. Only when seizures continue despite administration of a second bolus of magnesium sulphate should diazepam or thiopental be administered intravenously. ${ }^{[13]}$ In our study, the use of diazepam was not found to make any difference to the outcomes. The use of other anticonvulsants (phenytoin, thiopentone) was associated with an increase in the proportion of poor outcomes. In cases of seizures refractory to magnesium sulphate, it may therefore be safer to use a benzodiazepine.

According to Zeeman, most seizures occur during the intrapartum and postpartum periods. ${ }^{[21]}$ However, the present study confirmed Moodley and Daya's ${ }^{[7]} 1993$ findings, as in most cases the onset of seizures was antenatal. Mortality was higher among patients with onset of seizures intrapartum or postpartum. This would appear contrary to the findings of Mattar and Sibai, ${ }^{[22]}$ who suggested increased mortality if the onset of seizures was antepartum, but increased neurological morbidity among patients in whom seizure onset was postpartum. This may suggest a need for enhanced vigilance in the intra- and postpartum period. After discharge, we suggest that patients who have had HDP should have frequent appointments at their local clinic, and be given information about symptoms of severe pre-eclampsia that should prompt them to seek help early.

Primiparity is considered a risk factor for HDPs. ${ }^{[23]}$ However, in the present study, 
Table 4. Neurological findings: good outcome (neurologically intact) v. poor outcome (adverse neurological outcome or dead)*

\begin{tabular}{|c|c|c|c|c|}
\hline Variable & Total, $n$ & Good outcome, $n(\%)$ & Poor outcome, $n(\%)$ & $p$-value \\
\hline \multicolumn{5}{|c|}{ Eclampsia $(N=78)$} \\
\hline No & 26 & $22(84.6)$ & $4(15.4)$ & \multirow[t]{2}{*}{$0.027^{\dagger}$} \\
\hline Yes & 52 & $41(78.9)$ & $11(21.1)$ & \\
\hline \multicolumn{5}{|c|}{ Lowest recorded GCS $(N=76)$} \\
\hline$<10$ & 36 & $20(57.1)$ & $15(42.9)$ & \multirow[t]{2}{*}{$<0.001^{\dagger}$} \\
\hline$\geq 10$ & 40 & $36(94.7)$ & $2(5.3)$ & \\
\hline \multicolumn{5}{|c|}{ CT scan findings } \\
\hline \multicolumn{5}{|c|}{ CT scan: done $(N=77)$} \\
\hline No & 46 & $43(93.5)$ & $(6.5)$ & \multirow[t]{2}{*}{$<0.001^{+}$} \\
\hline Yes & 31 & $16(51.6)$ & $15(48.4)$ & \\
\hline \multicolumn{5}{|c|}{ Abnormal CT findings $(N=31)$} \\
\hline No & 12 & $8(66.7)$ & $4(33.3)$ & \multirow[t]{2}{*}{0.183} \\
\hline Yes & 19 & $8(42.1)$ & $11(57.9)$ & \\
\hline \multicolumn{5}{|c|}{ CT structural damage $(N=31)$} \\
\hline No & 21 & $13(61.9)$ & $8(38.1)$ & \multirow[t]{2}{*}{0.097} \\
\hline Yes & 10 & $3(30.0)$ & $7(70.0)$ & \\
\hline \multicolumn{5}{|c|}{ Emergency anticonvulsants } \\
\hline \multicolumn{5}{|c|}{ Magnesium sulphate (severe pre-eclampsia and eclampsia) $(N=78)$} \\
\hline No & 20 & $11(55.0)$ & $9(45.0)$ & \multirow[t]{2}{*}{$0.003^{+}$} \\
\hline Yes & 58 & $51(87.9)$ & $7(12.1)$ & \\
\hline \multicolumn{5}{|c|}{ Magnesium sulphate (eclampsia) $(N=50)$} \\
\hline No & 5 & $2(40.0)$ & $3(60.0)$ & \\
\hline Yes & 45 & $38(84.4)$ & $7(15.6)$ & $0.048^{\dagger}$ \\
\hline \multicolumn{5}{|c|}{ Benzodiazepines (eclampsia) $(N=50)$} \\
\hline No & 35 & $29(58)$ & $6(12)$ & \\
\hline Yes & 15 & $11(22)$ & $4(8)$ & 0.462 \\
\hline \multicolumn{5}{|c|}{ Other anticonvulsants (eclampsia) $(N=50)$} \\
\hline No & 45 & $38(84.4)$ & $7(15.6)$ & \\
\hline Yes & 5 & $2(40.0)$ & $3(60.0)$ & $0.048^{\dagger}$ \\
\hline $\begin{array}{l}\text { GCS }=\text { Glasg } \\
{ }^{*} N \text { varies as } \\
{ }^{\dagger} p=0.05 .\end{array}$ & & & & \\
\hline
\end{tabular}

primiparous patients had better outcomes, contrary to previous findings. ${ }^{[2]}$ Other risk factors for HDP, when analysed separately, did not make a significant difference to outcome.

Expectant management for HDP has been advocated as safe by some authorities. ${ }^{[13,24]}$ The results of this study suggest the need to exercise caution when managing such patients expectantly in under-resourced settings. We showed a statistically significant difference in the time (in weeks) of antenatal onset of hypertension between patients with poor outcomes (earlier onset) and those with good outcomes (later onset) (Table 2). According to von Dadelszen et al. ${ }^{[25]}$ the fullPIERS (Preeclampsia Integrated Estimate of RiSk) model can be used to identify patients who may or may not be eligible for expectant management. This model predicts adverse maternal outcome (maternal mortality or one or more serious central nervous system (CNS), cardiorespiratory, hepatic, renal or haematological morbidities) using predictors that include gestational age at eligibility, chest pain or dyspnoea, oxygen saturation, platelet count, serum creatinine and aspartate transaminase. None of these factors were found to significantly affect outcomes in our patients. The model is further limited by the fact that it was only validated for well-resourced settings. The new miniPIERS model aims to address that shortcoming by excluding laboratory variables, and a study to demonstrate its implementability (the Community-Level Interventions for Pre-eclampsia (CLIP) study) is underway. ${ }^{[26]}$

The rate of HIV infection was higher in the patients with poorer outcomes, and the seroprevalence among those with good outcomes was also notably lower (9.4\%) than the general antenatal seroprevalence of $38 \%{ }^{[27]}$ Several studies have attempted to determine whether or not there is an association between HIV and obstetric complications (including HDP), with conflicting results. A meta-analysis by Calvert and Ronsmans ${ }^{[28]}$ showed that HIV was associated with (non-proteinuric) pregnancy-induced hypertension (PIH), but not with pre-eclampsia or eclampsia. A recent re-analysis of local data on maternal mortality and HIV concurred with our present findings that there was less HIV among women with PIH, and furthermore that this lessened effect was reversed in women who accessed HIV treatment. ${ }^{[29]}$ A larger study is necessary to confirm the results. Pre-existing epilepsy also increased poor outcomes in our study ( $p=0.046)$; however, its effect on outcomes in HDP patients has not been widely studied.

The definitive treatment for HDP is delivery. ${ }^{[4]}$ Surgical delivery, when it was indicated, improved outcome among our patients in both analyses. This would indicate that the rate of surgery for HDP patients is appropriate in the institution.

This is the first study to evaluate the possible factors that contribute to maternal outcomes, especially mortality, as mentioned in the Saving Mothers Reports, ${ }^{[2,3]}$ and showed that most factors are only associated with, but not directly contributory to, poor outcomes. However, the low patient numbers and the retrospective nature of the study are limitations. Our most important recommendation is that there should be stricter adherence to protocol in the management of patients with HDP, including those in ICU. This recommendation is specified by a number of authorities. ${ }^{[2,3,12,30]}$ Specific factors to be attended to include earlier identification of patients needing high care and ICU referral 
Table 5. Critical care findings: Alive (neurologically intact or adverse neurological outcome) v. dead*

\begin{tabular}{|c|c|c|c|c|}
\hline Variable & Total, $n$ & Alive, $n(\%)$ & Died, $n(\%)$ & $p$-value \\
\hline \multicolumn{5}{|c|}{ Reason for admission to ICU/organ support } \\
\hline Airway $(N=85)$ & 80 & $70(87.5)$ & $10(12.5)$ & \\
\hline No & 5 & $5(100)$ & $0(0.0)$ & 0.400 \\
\hline \multicolumn{5}{|l|}{ Yes } \\
\hline \multicolumn{5}{|c|}{ Respiratory failure $(N=85)$} \\
\hline No & 61 & $57(93.4)$ & $4(6.6)$ & $0.027^{\dagger}$ \\
\hline Yes & 24 & $18(75.0)$ & $6(25.0)$ & \\
\hline \multicolumn{5}{|l|}{ LRTI $(N=79)$} \\
\hline No & 75 & $70(93.3)$ & $5(6.7)$ & $0.037^{\dagger}$ \\
\hline Yes & 4 & $2(50.0)$ & $2(50.0)$ & \\
\hline \multicolumn{5}{|c|}{ Cardiac failure/pulmonary oedema $(N=85)$} \\
\hline No & 75 & $65(86.7)$ & $10(13.3)$ & 0.219 \\
\hline Yes & 10 & $10(100.0)$ & $0(0.0)$ & \\
\hline \multicolumn{5}{|l|}{ Shock $(N=85)$} \\
\hline No & 71 & $61(85.9)$ & $10(14.1)$ & 0.135 \\
\hline Yes & 14 & $14(100)$ & $0(0.0)$ & \\
\hline \multicolumn{5}{|c|}{ Neuro-protection $(N=85)$} \\
\hline No & 42 & $37(88.1)$ & $5(11.9)$ & 0.968 \\
\hline Yes & 43 & $38(88.4)$ & $5(11.6)$ & \\
\hline \multicolumn{5}{|c|}{ Renal replacement therapy $(N=85)$} \\
\hline No & 82 & $72(87.8)$ & $10(12.2)$ & 0.520 \\
\hline Yes & 3 & $3(100)$ & $0(0.0)$ & \\
\hline \multicolumn{5}{|c|}{ Number of organ failures $(N=85)$} \\
\hline 0 & 3 & $3(100)$ & $0(0)$ & 0.565 \\
\hline 1 & 60 & $54(90.0)$ & $6(10.0)$ & \\
\hline 2 & 21 & $17(81.0)$ & $4(19.0)$ & \\
\hline 3 & 1 & $1(100)$ & $0(0.0)$ & \\
\hline
\end{tabular}

Table 6. Critical care findings: good outcome (neurologically intact) vs. poor outcome (adverse neurological outcome or dead)

\begin{tabular}{|c|c|c|c|c|}
\hline Variable & Total, $n$ & Good outcome, $n(\%)$ & Poor outcome, $n(\%)$ & $p$-value \\
\hline \multicolumn{5}{|c|}{ Reason for admission to ICU/organ support } \\
\hline \multicolumn{5}{|l|}{ Airway $(N=82)$} \\
\hline No & 78 & $60(76.9)$ & $18(23.1)$ & 0.277 \\
\hline Yes & 4 & $4(100.0)$ & $0(0.0)$ & \\
\hline \multicolumn{5}{|l|}{ Respiratory failure $(N=82)$} \\
\hline No & 58 & $47(81.0)$ & $11(19.0)$ & 0.31 \\
\hline Yes & 24 & $17(70.8)$ & $7(29.2)$ & \\
\hline \multicolumn{5}{|l|}{ LRTI $(N=76)$} \\
\hline No & 72 & $59(81.9)$ & $13(19.1)$ & 0.118 \\
\hline Yes & 4 & $2(50.0)$ & $2(50.0)$ & \\
\hline \multicolumn{5}{|c|}{ Cardiac failure/pulmonary oedema $(N=82)$} \\
\hline No & 72 & $54(75.0)$ & $18(25.0)$ & 0.073 \\
\hline Yes & 10 & $10(100.0)$ & $0(0.0)$ & \\
\hline Shock $(N=82)$ & 68 & $52(76.5)$ & $16(23.5)$ & 0.447 \\
\hline No & 14 & $12(85.7)$ & $2(14.3)$ & \\
\hline \multicolumn{5}{|l|}{ Yes } \\
\hline Neuro-protection $(N=82)$ & 42 & $35(83.3)$ & $7(16.7)$ & 0.236 \\
\hline No & 40 & $29(72.5)$ & $11(17.5)$ & \\
\hline \multicolumn{5}{|l|}{ Yes } \\
\hline \multicolumn{5}{|c|}{ Renal replacement therapy $(N=82)$} \\
\hline No & 79 & $62(78.5)$ & $17(21.5)$ & 0.627 \\
\hline Yes & 3 & $2(66.7)$ & $1(33.3)$ & \\
\hline \multicolumn{5}{|c|}{ Number of organ failures $(N=82)$} \\
\hline 0 & 3 & $3(100.0)$ & $0(0.0)$ & 0.468 \\
\hline 1 & 57 & $46(80.7)$ & $11(19.3)$ & \\
\hline 2 & 21 & $14(66.7)$ & $7(33.3)$ & \\
\hline 3 & 1 & $1(100.0)$ & $0(0.0)$ & \\
\hline
\end{tabular}


(possibly including the use of scoring systems such as the fullPIERS or miniPIERS scores in obstetrics wards), paying closer attention to the CNS, ensuring the appropriate use of magnesium sulphate, closer monitoring of patients with HDP intra- and postpartum and closer attention to the management of HIV and respiratory infection.

\section{Conclusion}

The findings of this study show that the mortality rate for HDP had not decreased over a decade in ICU, despite available recommendations. This indicates the need for appropriate action. Most notably, this must involve stricter implementation of a protocol for the management of HDP patients. Attention needs to be paid to the factors suggesting poor outcome in this study, especially lowest GCS score $<10$ and failure to use magnesium sulphate when indicated. Further studies should focus on the effectiveness of the protocol to be implemented. Moreover, the reasons for the decrease in the number of HDP patients presenting to the ICU in this period compared with the year 2000 should be determined, including the potential impact of prophylactic calcium supplementation in antenatal patients.

Acknowledgements. The authors acknowledge the help rendered by Prof. G Matthews (Department of Applied Mathematics, University of KwaZulu-Natal (UKZN)) and Mr B Tlou (Biostatistics Department, UKZN) with regard to the statistical analysis. The KZN Department of Health, King Edward VIII Medical Records, Mr S Pershad (Head of Clinical Unit, ICU, Inkosi Albert Luthuli Central Hospital) and Mr DJ Tuffnell (Yorkshire Obstetrics Critical Care Group) are further acknowledged for their ready assistance in some aspects of the production of this article. Dr C Tiloke assisted in the manuscript preparations.

Author contributions. SG was responsible for study design, data collection, analysis and writing of the manuscript. MS assisted with study design and manuscript preparations. KdV assisted with data analysis and manuscript preparations. All authors read and approved the manuscript.

Funding. None.

Conflicts of interest. None.

1. Say L, Chou D, Gemmill A, et al. Global causes of maternal death: A WHO systematic analysis. Lancet Glob Health 2014;2(6):e323-e333. https://doi.org/10.1016/S2214-109X(14)70227-X

2. National Committee for Confidential Enquiry into Maternal Deaths (South Africa). Saving Mothers 2008 - 2010 short report. Pretoria: NCCEMD, 2011. http://www.health.gov.za/index. php/2014-08-15-12-55-04/category/100-2012rp?download=189:saving-mothers-2008-2010fifth-report-on-the-confidential-enquiries-into-maternal-deaths-in-south-africa-short-report (accessed 12 June 2012)

3. National Committee for Confidential Enquiry into Maternal Deaths (South Africa). Saving Mothers 2011 - 2013: Sixth Report on the Confidential Enquiries into Maternal Deaths in South Africa. Pretoria: NCCEMD, 2014. http://www.kznhealth.gov.za/mcwh/Maternal/SavingMothers-2011-2013-short-report.pdf (accessed 28 September 2015). 4. Brown MA. Diagnosis and classification of preeclampsia and other hypertensive disorders of
pregnancy. In: Belfort MA, Thornton S, Saade GR, eds. Hypertension in Pregnancy. New York: Marcel Dekker, Inc., 2003:1-16.

5. Tranquilli AL, Dekker G, Magee L, et al. The classification, diagnosis and management of the hypertensive disorders of pregnancy: A revised statement from the ISSHP. Pregnancy Hypertens 2014;4(2):97-104. https://doi.org/10.1016/j.preghy.2014.02.001
6. Linton DM, Anthony J. Critical care management of severe pre-eclampsia. Intensive Care Med 1997;23(3):248-255. https://doi.org/10.1007/s001340050324

7. Moodley J, Daya P. Eclampsia: A continuing problem in developing countries. Int J Gynaecol Obstet 1994;44(1):9-14. https://doi.org/10.1016/0020-7292(94)90016-7

8. Mahutte NG, Murphy-Kaulbeck L, Le Q, Solomon J, Benjamin A, Boyd ME. Obstetric admissions to the intensive care unit. Obstet Gynecol 1999;94(2):263-266. https://doi.org/10.1016/S00297844(99)00274-4

9. Bhagwanjee S, Paruk F, Moodley J, Muckart DJJ. Intensive care unit morbidity and mortality from eclampsia: An evaluation of the Acute Physiology and Chronic Health Evaluation II score and the Glasgow Coma Scale score. Crit Care Med 2000;28(1):120-124. https://doi.org/10.1097/00003246200001000-00020

10. Neto AFO, Parpinelli MA, Cecatti JG, Souza JP, Sousa MH. Factors associated with maternal death in women admitted to an intensive care unit with severe maternal morbidity. Int $J$ Gynaecol Obstet 2009;105(3):252-256. https://doi.org/10.1016/j.ijgo.2009.01.025

11. Mantel GD, Buchmann E, Rees H, Pattinson RC. Severe acute maternal morbidity: A pilot study of a definition for a near-miss. BJOG 1998;105(9):985-990. https://doi.org/10.1111/j.1471-0528.1998 tb10262.x

12. Baskett TF. Epidemiology of obstetric critical care. Best Pract Res Clin Obstet Gynaecol 2008;22(5):763-774. https://doi.org/10.1016/j.bpobgyn.2008.06.002

13. Zeeman GG. Neurologic complications of pre-eclampsia. Semin Perinatol 2009;33(3):166-172 https://doi.org/10.1053/j.semperi.2009.02.003

14. Price LC, Slack A, Nelson-Piercy C. Aims of obstetric critical care management. Best Pract Res Clin Obstet Gynaecol 2008;22(5):775-799. https://doi.org/10.1016/.bpobgyn.2008.06.001

15. Rout CC. Anaesthesia and analgesia for the critically ill parturient. Best Pract Res Clin Obstet Gynaecol 2001;15(4):507-522. https://doi.org/10.1053/beog.2001.0197

16. Servillo G, Bifulco F, de Robertis E, et al. Posterior reversible encephalopathy syndrome in intensive care medicine. Intensive Care Med 2007;33(2):230-236. https://doi.org/10.1007/s00134006-0459-0

17. Heaviside V, Hayes M. Cerebral protection. In: Bernstein AD, Soni N, eds. Oh’s Intensive Care Manual. Oxford: Butterworth Heinemann (Elsevier), 2009:563-574

18. The Eclampsia Trial Collaborative Group. Which anti-convulsant for women with eclampsia? Evidence from the Collaborative Eclampsia Trial. Lancet 1995;345(8963):1455-1463. https://doi. org/10.1016/S0140-6736(95)91034-4

19. Dubé L, Granry J. The therapeutic use of magnesium in anesthesiology, intensive care and emergency medicine: A review. Can J Anaesth 2003;50(7):732-746. https://doi.org/10.1007/ BF03018719

20. The Magpie Trial Collaborative Group. Do women with pre-eclampsia, and their babies, benefit from magnesium sulphate? The Magpie Trial: A randomised placebo-controlled trial. Lancet 2002;359(9321):1877-1890. https://doi.org/10.1016/S0140-6736(02)08778-0

21. Zeeman GG. Obstetric critical care: A blueprint for improved outcome. Crit Care Med 2006;34(9): S208-S214. https://doi.org/10.1097/01.CCM.0000231884.99763.69

22. Mattar F, Sibai BM. Eclampsia: VIII. Risk factors for maternal morbidity. Am J Obstet Gynecol 2000;182(2):307-312. https://doi.org/10.1016/S0002-9378(00)70216-X

23. American College of Obstetricians and Gynecologists. Hypertension in pregnancy. Report of the American College of Obstetricians and Gynecologists' task force on hypertension in pregnancy. Obstet Gynecol 2013;122(5):1122-1131. https://doi.org/10.1097/01.AOG.0000437382.03963.88

24. Oettle C, Hall D, Roux A, Grove D. Early onset severe pre-eclampsia: Expectant management at a secondary hospital in close association with a tertiary institution. BJOG 2005;112(1):84-88. https://doi.org/10.1111/j.1471-0528.2004.00262.x

25. Von Dadelszen P, Payne B, Jing L, et al., for the PIERS Study Group. Prediction of adverse maternal outcomes in pre-eclampsia: Development and validation of the fullPIERS model. Lancet 2011;377(9761):219-227. https://doi.org/10.1016/S0140-6736(10)61351-7

26. Payne BA, Hutcheon JA, Ansermino JM, et al., for the miniPIERS Study Working Group. A Risk prediction model for the assessment and triage of women with hypertensive disorders of pregnancy in low-resourced settings: The miniPIERS (Pre-eclampsia Integrated Estimate of RiSk) pregnancy in low-resourced settings: The miniPIERS (Pre-eclampsia Integrated Estimate of RiSk)
multi-country prospective cohort study. PLoS Med 2014;11(1):e1001589. https://doi.org/10.1371 multi-country prospective

27. National Department of Health, South Africa. The 2015 national antenatal sentinel HIV \& syphilis survey. Pretoria: NDoH, 2017. http://www.health.gov.za/index.php/shortcodes/201503-29-10-42-47/2015-04-3008-18-10/2015-04-30-08-21-56?download=2584:2015-nationalantenatal-hiv-prevalence-survey-final-23oct17 (accessed 20 December 2018).

28. Calvert C, Ronsmans C. HIV and the risk of direct obstetric complications: A systematic review and meta-analysis. PLoS One 2013;8(10):e74848. https://doi.org/10.1371/journal.pone.0074848

29. Sebitloane HM, Moodley J, Sartorius B. Associations between HIV, highly active anti-retroviral therapy and hypertensive disorders of pregnancy among maternal deaths in South Africa 2011 - 2013. Int J Gynaecol Obstet 2017;136(2):195-199. https://doi.org/10.1002/ijgo.12038

30. Tuffnell DJ, Jankowicz D, Lindow SW, et al. Outcomes of severe pre-eclampsia/eclampsia in Yorkshire 1999/2003. BJOG 2005;112(7):875-880. https://doi.org/10.1111/j.14710528.2005.00565.x 